\title{
Contaminated Heparin Associated with Adverse Clinical Events and Activation of the Contact System
}

Takashi Kei Kishimoto, Ph.D., Karthik Viswanathan, Ph.D., Tanmoy Ganguly, Ph.D., Subbiah Elankumaran, Ph.D., Sean Smith, B.S., Kevin Pelzer, Ph.D., Jonathan C. Lansing, Ph.D., Nammalwar Sriranganathan, Ph.D., Ganlin Zhao, M.D., Zoya Galcheva-Gargova, Ph.D., Ali Al-Hakim, Ph.D., Gregory Scott Bailey, B.S., Blair Fraser, Ph.D., Sucharita Roy, Ph.D., Thomas Rogers-Cotrone, M.S., Lucinda Buhse, Ph.D., Mark Whary, Ph.D., James Fox, Ph.D., Moheb Nasr, Ph.D., Gerald J. Dal Pan, M.D., Zachary Shriver, Ph.D., Robert S. Langer, Sc.D., Ganesh Venkataraman, Ph.D., K. Frank Austen, M.D., Janet Woodcock, M.D., and Ram Sasisekharan, Ph.D.

Momenta Pharmaceuticals (T.K.K., T.G., S.S., J.C.L., G.Z., Z.G.-G., G.S.B., S.R., Z.S., G.V.), the Harvard-Massachusetts Institute of Technology Division of Health Sciences and Technology, Koch Institute for Integrative Cancer Research (K.V., Z.S., R.S.L., G.V., R.S.), and the Massachusetts Institute of Technology (M.W., J.F.) - all in Cambridge, MA; Virginia-Maryland Regional College of Veterinary Medicine, Virginia Polytechnic Institute and State University, Blacksburg (S.E., K.P., N.S., T.R.-C.); the Center for Drug Evaluation and Research, Food and Drug Administration, Silver Spring, MD (A.A.-H., B.F., L.B., M.N., G.J.D.P., J.W.); and Brigham and Women's Hospital and Harvard Medical School, Boston (K.F.A.)

\section{Abstract}

Background-There is an urgent need to determine whether oversulfated chondroitin sulfate (OSCS), a compound contaminating heparin supplies worldwide, is the cause of the severe anaphylactoid reactions that have occurred after intravenous heparin administration in the United States and Germany.

Methods-Heparin procured from the Food and Drug Administration, consisting of suspect lots of heparin associated with the clinical events as well as control lots of heparin, were screened in a blinded fashion both for the presence of OSCS and for any biologic activity that could potentially link the contaminant to the observed clinical adverse events. In vitro assays for the activation of the contact system and the complement cascade were performed. In addition, the ability of OSCS to recapitulate key clinical manifestations in vivo was tested in swine.

Results-The OSCS found in contaminated lots of unfractionated heparin, as well as a synthetically generated OSCS reference standard, directly activated the kinin-kallikrein pathway in human plasma, which can lead to the generation of bradykinin, a potent vasoactive mediator. In addition, OSCS induced generation of C3a and C5a, potent anaphylatoxins derived from complement proteins. Activation of these two pathways was unexpectedly linked and dependent on fluid-phase activation of factor XII. Screening of plasma samples from various species indicated that swine and humans are sensitive to the effects of OSCS in a similar manner. OSCScontaining heparin and synthetically derived OSCS induced hypotension associated with kallikrein activation when administered by intravenous infusion in swine.

Copyright $(2008$ Massachusetts Medical Society.

Address reprint requests to Dr. Sasisekharan at the Department of Biological Engineering, Harvard-MIT Division of Health Sciences and Technology, Koch Institute for Integrative Cancer Research, Massachusetts Institute of Technology, 77 Massachusetts Ave., 16-561, Cambridge, MA 02139, or at rams@ mit.edu.

No other potential conflict of interest relevant to this article was reported. 
Conclusions-Our results provide a scientific rationale for a potential biologic link between the presence of OSCS in suspect lots of heparin and the observed clinical adverse events. An assay to assess the amidolytic activity of kallikrein can supplement analytic tests to protect the heparin supply chain by screening for OSCS and other highly sulfated polysaccharide contaminants of heparin that can activate the contact system.

In january 2008, health authorities in the United States began receiving reports of clusters of acute hypersensitivity reactions in patients undergoing dialysis that had been occurring since November 2007. Symptoms included hypotension, facial swelling, tachycardia, urticaria, and nausea. Although initial investigations focused on dialysis equipment, an investigation by the Centers for Disease Control and Prevention identified the receipt of heparin sodium for injection (1000 U per milliliter, in 10-ml and 30-ml multidose vials), manufactured by Baxter Healthcare, as a common feature of the cases. ${ }^{1}$ This finding led Baxter Healthcare to recall, on January 17, 2008, nine lots of heparin sodium for injection. As of April 13, 2008, there were 81 reports of death that involved at least one sign or symptom of an allergic reaction or hypotension in patients receiving heparin since January 1, 2007. However, the fact that allergic symptoms or hypotension were reported does not mean that these were the cause of death in all cases.

After this initial recall, there were continuing reports of allergic-type reactions, including some deaths, after injection of bolus heparin not only in patients undergoing dialysis but also in patients in other clinical settings, such as those undergoing cardiac procedures. On February 28, 2008, Baxter Healthcare recalled all remaining lots and doses of its multidose and single-dose vials of heparin sodium for injection and HEP-LOCK heparin flush products. Since that recall, monitoring by the Food and Drug Administration (FDA) has indicated that, in March 2008, the number of deaths reported in association with heparin usage had returned to baseline levels. ${ }^{2}$

However, on March 6, a heparin recall was announced in Germany because of a cluster of reactions in patients undergoing dialysis that were linked to a different manufacturer's heparin. On the same day, the FDA posted descriptions of analytic methods on its Web site and recommended that all manufacturers and regulatory authorities screen for a contaminant in heparin. ${ }^{3}$ This screening revealed widespread contamination of the heparin supply in at least 12 countries.

The contaminant was recently identified as an unusual oversulfated form of chondroitin sulfate (OSCS) representing up to approximately $30 \% \mathrm{wt} / \mathrm{wt}$ in suspect lots of heparin; no other contaminants were observed. ${ }^{4}$ In addition, dermatan sulfate, a known impurity of heparin, was found in selected samples. Both heparin and chondroitin sulfate are members of the glycosaminoglycan family of complex polysaccharides; heparin contains a disaccharide repeat unit of glucuronic-iduronic acid linked to glucosamine, and chondroitin sulfate contains a disaccharide repeat unit of glucuronic acid linked to galactosamine. Analysis of the contaminant unexpectedly revealed an unusual type of sulfation not found in any natural sources of chondroitin sulfate and indicated that OSCS, containing four sulfates per disaccharide unit, is structurally similar to heparin (see the Supplementary Appendix, available with the full text of this article at www.nejm.org).

However, the biologic link between the presence of the OSCS in heparin and the adverse clinical events remained to be established. Highly charged polyanionic polymers are known to modulate various enzymatic cascades in plasma, affecting coagulation, fibrinolysis, inflammation, and vasculature function. ${ }^{5,6}$ Bradykinin, a potent vasoactive peptide mediator, is generated through the activation of the contact system of coagulation, which is initiated upon contact of factor XII with a negatively charged surface in the presence of prekallikrein 
and high-molecular-weight kininogen. Highly sulfated polysaccharides have been shown to serve as a negatively charged surface that can initiate fluid-phase activation of the contact system. ${ }^{5,7}$ However, initial attempts to recapitulate the adverse responses in experimental models were unsuccessful. ${ }^{8}$ Without a definitive link between the contaminant and the clinical reactions, concerns remain that the screening tests currently in place may not be adequate to prevent further cases. We therefore set out to identify a biologic basis for a link between OSCS and allergic-type reactions.

\section{Case Report}

A representative case involved a 63-year-old woman with a complex medical history, including end-stage renal disease treated with the use of hemodialysis for 7 years, who received heparin intravenously during hemodialysis (5000-U loading dose and $500 \mathrm{U}$ per hour during the procedure) three times weekly. In mid-January 2008, the development of "low blood pressure" was reported, along with nausea and dyspnea, during dialysis. She was treated with normal saline and oxygen ( 2 liters per minute), and the rates of ultrafiltration and blood flow were slowed. She recovered after 30 minutes, and dialysis was continued. Two days later, she again received intravenous heparin (5000-U loading dose and $500 \mathrm{U}$ per hour) from the same lots of heparin from the same manufacturer (Baxter Healthcare). Immediately after dialysis was initiated, the patient had an anaphylactoid reaction, with a sudden drop in blood pressure (to 65/34 mm Hg), dyspnea, nausea, vomiting, and constitutional symptoms. She was treated with a bolus of normal saline and oxygen ( 2 liters per minute). Hemodialysis was continued for another hour. The patient continued to feel ill, was admitted to the hospital, and was discharged 2 days later, after recovery. Further dialysis was performed with the use of heparin from another manufacturer.

\section{Methods}

\section{Test Samples}

Twenty-nine clinical lots of heparin, including 13 associated with clinical adverse events, were procured from the FDA and coded as unknown samples 1 through 29. A laboratory lot of heparin was included as a control. For all analytic and biologic tests, samples were dosed on a weight basis; specific activity of heparin is typically approximately $180 \mathrm{U}$ per milligram. OSCS was purified to homogeneity from a lot of heparin that was known to be contaminated, as previously described. ${ }^{4}$ Briefly, OSCS-contaminated heparin was subjected to anion-exchange chromatography followed by alcohol precipitation to isolate the contaminant. ${ }^{4}$ The identity of the contaminant was confirmed by means of multiple orthogonal techniques, including multidimensional nuclear magnetic resonance (NMR), enzymatic digestion followed by high-performance liquid chromatography, and liquid chromatography-mass spectrometry. ${ }^{4}$ After identification of the contaminant as OSCS, a synthetic standard was generated through chemical sulfonation of chondroitin sulfate A and was exhaustively characterized to ensure authenticity, as previously described. ${ }^{4}$ The synthetic OSCS was used in spiking experiments to qualify the analytic procedures (especially one-dimensional proton NMR, described below) to determine limits of detection and to establish accurate quantification. ${ }^{4}$ The limit of detection for this assay was determined to be $0.3 \%$ on a weight basis for both dermatan sulfate and OSCS.

\section{Analytic Methods}

To ensure accurate identification and quantification of any contaminants and impurities, the 29 coded test samples were subjected to orthogonal analytic techniques. Proton NMR, anion-exchange chromatography, and capillary electrophoresis were used to screen the samples for the presence of OSCS, dermatan sulfate, and other nonheparin components. The 
levels of OSCS and dermatan sulfate were quantified with the use of a 600-MHz NMR instrument to ensure peak resolution. The details of quantification, as well as a representative spectrum, are given in Figure 1 and Table 1 in the Supplementary Appendix. For samples with unusual patterns, the identity of contaminants or impurities, including OSCS, was confirmed by means of detailed characterization, including the use of multidimensional NMR. ${ }^{4}$

\section{Amidolytic Activity of Kallikrein}

Pooled human plasma or factor XII-depleted plasma (American Diagnostica) was treated with various concentrations of coded test samples of heparin, chondroitin sulfate A, or synthetic OSCS for 5 minutes at $37^{\circ} \mathrm{C}$. The amidolytic activity of kallikrein (with a small contribution of factor XII) $)^{9}$ was assessed by adding the S-2302 chromagenic substrate (DPro-Phe-Arg-p-nitroaniline [pNA]) for 30 minutes at $37^{\circ} \mathrm{C}$, followed by spectrophotometric measurement of the absorbance at $450 \mathrm{nM}$.

\section{Generation of $\mathrm{C} 3 \mathrm{a}$ and $\mathrm{C} 5 \mathrm{a}$}

Pooled human EDTA plasma or factor XII-depleted plasma (American Diagnostica) was treated with various concentrations of OSCS-contaminated heparin, control heparin, chondroitin sulfate A, or synthetic OSCS for 30 minutes at $37^{\circ} \mathrm{C}$. C3a and C5a activation products of the complement cascade were assayed by means of a sandwich enzyme-linked immunosorbent assay (ELISA), as specified in the manufacturer's instructions (Becton Dickinson and Integrated Biotech Laboratories for C3a and C5a, respectively).

\section{In Vivo Studies}

The swine were handled and treated in compliance with the Public Health Service Policy on Humane Care and Use of Laboratory Animals and the federal Animal Welfare Act. The experimental procedures were performed according to the Institutional Animal Care and Use Committee- approved protocol of the Virginia Polytechnic Institute and State University, Blacksburg. Domestic Yorkshire crossbred swine were of either sex (Virginia Polytechnic Institute and State University) and ranged in weight from 10 to $25 \mathrm{~kg}$. They were initially anesthetized with an intravenous injection of $6 \mathrm{mg}$ of tiletamine hydrochloride per kilogram of body weight and $2.2 \mathrm{mg}$ of xylazine per kilogram, and then a single-lumen silicone catheter was implanted in the left jugular vein of each animal. Adequate anesthesia was maintained throughout the procedure with the administration of supplemental tiletamine. After a 5-minute stabilization period, each pig received an intravenous bolus infusion of 5 $\mathrm{mg}$ of the test substance per kilogram (three to six pigs per test substance). All the pigs were continuously monitored for vital signs with the use of an oscillometric blood pressure monitor (Cardell 9401/9403, CAS Medical Systems) for systolic, diastolic, and mean arterial blood pressures, pulse oximetry for pulse and respiratory rates, and a rectal probe for body temperature. At the end of the 60-minute observation period, the animals were euthanized with the use of an intravenous infusion of Fatal Plus (Vortech Pharmaceuticals) at a dose of $0.22 \mathrm{ml}$ per kilogram. Blood samples were collected at baseline and at 5, 10, 20, 40, and 60 minutes and were kept in $5 \mathrm{mM}$ EDTA. Plasma was isolated after centrifugation at $4^{\circ} \mathrm{C}$ and flash-frozen on dry ice. Frozen samples were thawed at $4^{\circ} \mathrm{C}$ and assayed for amidolytic activity of kallikrein with the addition of the S-2302 chromagenic substrate (D-Pro-Phe-ArgpNA), as described above.

\section{Results}

Given the association of activation of the contact system with negatively charged polysaccharides, we sought to elucidate whether an in vitro biologic response could be correlated with the identity or levels of contaminant within heparin lots. To this end, we 
examined the ability of a sample of OSCS-contaminated heparin, containing 19.3\% wt/wt OSCS (Table 1 in the Supplementary Appendix), to activate kallikrein amidolytic activity in human plasma (Fig. 1A). The contaminated heparin showed a bell-shaped dose response, which is typical of glycosaminoglycan-mediated responses. ${ }^{5,10}$ At 2.5 and $25 \mu \mathrm{g}$ per milliliter, robust activation of kallikrein was found with the contaminated heparin sample but not with a control sample of uncontaminated heparin. These concentrations are in the range of a clinically efficacious concentration of heparin of approximately 1 to $5 \mu \mathrm{g}$ per milliliter, based on a specific activity of about $180 \mathrm{U}$ per milligram. High concentrations of the OSCS-contaminated heparin ( $250 \mu \mathrm{g}$ per milliliter) induced little or no amidolytic activity of kallikrein, suggesting that at this concentration, heparin may inhibit or cause depletion of factor XII, as previously described. 7,11,12 This high concentration of heparin also prevented activation of the contact system in response to kaolin, a potent activator (data not shown).

To further verify that the contaminant was responsible for the activation of the contact system, OSCS was purified to homogeneity by means of anion-exchange chromatography followed by alcohol precipitation. In addition, an OSCS standard was created through chemical sulfonation of chondroitin sulfate A, to form OSCS. ${ }^{4}$ The purified contaminant and the OSCS standard were identical, as judged by several orthogonal analytic techniques, including two-dimensional NMR. ${ }^{4}$ Both the purified contaminant and the synthetic OSCS showed robust activation of kallikrein activity at $0.25 \mu \mathrm{g}$ and $2.5 \mu \mathrm{g}$ per milliliter (Fig. 1A). The peak activity of the purified contaminant and the synthetic OSCS standard were observed at a level that was approximately an order of magnitude lower than that found for the contaminated heparin sample. This is consistent with the observation that the OSCS constituted approximately $20 \%$ of the contaminated sample. Chondroitin sulfate A showed no induction of amidolytic activity.

These results are in good agreement with the observations of Hojima et al., ${ }^{5}$ who demonstrated that oversulfated chondroitin, but not chondroitin A, B, or C, can activate the kinin pathway. Heparin also activated the contact system in an in vitro model system involving purified protein components ${ }^{5,13}$ but did not in plasma, ${ }^{13}$ suggesting that negativeregulatory factors present in plasma may prevent activation of the contact system by heparin. One such mechanism is the fact that heparin is known to enhance antithrombin IIImediated inhibition of factor XII. Our results indicate that OSCS, in contrast to heparin but similar to dextran sulfate, ${ }^{13}$ can activate the contact system in plasma.

The 29 heparin samples procured from the FDA, consisting of both suspect heparin lots associated with clinical events as well as control heparin lots, were screened in a blinded fashion for both the presence of OSCS and the ability to activate the contact system (Fig. 1B). There was complete correspondence between the presence of detectable amounts of OSCS by one-dimensional proton NMR and the ability of a sample to induce robust amidolytic activity of kallikrein (Fig. 1B). The biologic activity was generally characterized as an all-or-none response, with all 13 samples containing detectable levels of OSCS having a positive response at $25 \mu \mathrm{g}$ or $2.5 \mu \mathrm{g}$ per milliliter. Sample 11, which contained the highest level of contaminant (27.4\%), also showed activity at $0.25 \mu \mathrm{g}$ per milliliter, whereas Sample 25 , which contained the lowest level of contaminant (2.4\%), showed only modest activity at $2.5 \mu \mathrm{g}$ per milliliter. In contrast, there was no association between the level of inducible kallikrein activity and the level of dermatan sulfate (Fig. 2 in the Supplementary Appendix), an impurity found in many heparin preparations.

Direct activation of the contact system by the contaminated heparin and the synthetic OSCS standard was confirmed through the use of human plasma depleted of factor XII, the upstream activator of prekallikrein ${ }^{14}$ (Fig. 2). The contaminated heparin, the synthetically 
derived OSCS, and the positive control (the kaolin-containing reagent) all failed to induce the amidolytic activity of kallikrein in factor XII-deficient plasma.

We next examined the ability of contaminated heparin to generate C3a and C5a, potent anaphylatoxins derived from complement proteins Expo sure of human plasma to the contaminated heparin, but not to control heparin, induced the production of C5a (Fig. 3).

OSCS-induced C5a generation showed a bell-shaped dose response similar to that found for kallikrein activation. Peak C5a activity was observed at $50 \mu \mathrm{g}$ and $5 \mu \mathrm{g}$ per milliliter of heparin containing $19.3 \%$ OSCS. At $500 \mu \mathrm{g}$ per milliliter, significant generation of C5a was not observed. Similar results were obtained with the purified OSCS isolated from contaminated heparin and the synthetic OSCS standard, but not with chondroitin sulfate A.

Surprisingly, the generation of C5a by OSCS-contaminated heparin was more robust in the presence of EDTA, a $\mathrm{Ca}^{2+}$ - and $\mathrm{Mg}^{2+}$-chelating agent, than in the absence of EDTA. The classic and alternative pathways of complement activation are known to be dependent upon $\mathrm{Ca}^{2+}$ and $\mathrm{Mg}^{2+}$, respectively. As expected, EDTA blocked C3a and C5a generation in response to zymosan, a potent activator of the alternative pathway (Fig. 4). These results suggested the possibility that OSCS induces the generation of C3a and C5a in a manner that bypasses the $\mathrm{C} 3$ and $\mathrm{C} 5$ convertases. To determine whether the generation of $\mathrm{C} 3 \mathrm{a}$ and $\mathrm{C} 5 \mathrm{a}$ was linked to the activation of the contact system, we next examined C3a and C5a generation in factor XII-depleted plasma (Fig. 4). As expected, zymosan induced the generation of C3a and C5a in factor XII-depleted plasma, and this activity was inhibited by EDTA. In contrast, neither C3a nor C5a was generated in factor XII- depleted plasma activated with OSCS, suggesting that OSCS bypasses the normal pathways for complement activation in a manner that is dependent on contact activation through factor XII. The generation of C5a could be restored by reconstituting the factor XII-depleted plasma with purified factor XII (Fig. 4A). This finding is further supported by the observation that C5a generation induced by OSCS-contaminated heparin can be inhibited by aprotinin, a protease inhibitor of kallikrein but not of factor XIIa (Fig. 4A). Crosstalk between the contact system and the complement cascade has been suggested previously. ${ }^{15-18}$ For example, factor XII has been shown to activate the classical pathway by activating $\mathrm{C} 1 .{ }^{15} \mathrm{It}$ has also been proposed to substitute for factor $\mathrm{D}$ in activating the alternative pathway. ${ }^{16}$ However, in these cases, activation of the complement cascade still occurs through divalent cation-dependent pathways. Kallikrein has been shown to act directly on C5 to generate C5a-like biologic activity. ${ }^{17}$ Both kallikrein and factor XII can activate the plasminogen pathway leading to the activation of plasmin, which has also been implicated in complement activation. ${ }^{18}$ Preliminary data suggest that OSCS is unable to induce C5a generation in plasminogendepleted plasma (data not shown).

To identify an appropriate species for in vivo testing of OSCS, a panel of plasma samples were screened for amidolytic activity in response to OSCS-contaminated heparin (Fig. 5A). Only swine plasma supported robust amidolytic activity of kallikrein in response to kaolin and OSCS-contaminated heparin but not control heparin. In contrast, rabbit, horse, and rat plasma showed moderate-to-robust amidolytic activity in response to kaolin but not to OSCS-contaminated heparin. These findings are consistent with a report that initial attempts to provoke an allergic response with suspect lots of heparin were unsuccessful. ${ }^{8}$ Similarly, we found that rabbits treated with $5 \mathrm{mg}$ of intravenous OSCS-contaminated heparin per kilogram showed no change in temperature, blood pressure, or heart rate as compared with rabbits treated with control heparin (data not shown). Wiggins ${ }^{19}$ demonstrated previously that dextran sulfate can induce hypotension in rabbits, but only at a high dose (20 mg per kilogram) and in a manner independent of complement or kinin activation. In contrast, moderate doses of dextran sulfate ( $5 \mathrm{mg}$ per kilogram) induced a robust hypotensive response in pigs that was dependent on activation of the contact system. ${ }^{20}$ 
To test the in vivo activity of OSCS, pigs were treated with a single intravenous dose (5 mg per kilogram) of OSCS-contaminated heparin, control heparin, synthetic OSCS, or chondroitin sulfate A and were monitored for 60 minutes. Animals treated with control heparin and those treated with OSCS-contaminated heparin showed similar anti-Xa activity during the entire 60-minute observation period (activity at 5 minutes, approximately 3 to 4 IU per milliliter) (Fig. 4 in the Supplementary Appendix). Animals treated with chondroitin sulfate A or synthetic OSCS showed no anti-Xa activity. These results suggest that any anticoagulant activity of OSCS is mediated through a non-antithrombin III-dependent mechanism. Two of six animals treated with OSCS-contaminated heparin had at least a 30\% drop in blood pressure over the first 30 minutes after infusion (Fig. 5B). One animal remained in a hypotensive state for more than 15 minutes. In contrast, none of the four animals treated with control heparin showed any substantive changes in blood pressure. The adverse events were more severe in pigs treated with the synthetic OSCS, a result consistent with the greater exposure to OSCS in animals treated with pure OSCS as compared with contaminated heparin containing approximately 20 to $30 \%$ OSCS. All three pigs treated with synthetic OSCS showed a profound drop in blood pressure (maximal decrease, 45 to 59\%) and a concurrent increase in heart rate within minutes after infusion. One animal had difficulty breathing and became cyanotic after a precipitous drop in blood pressure. The heart rate of a second animal increased from 114 beats per minute to 196 beats per minute within 4 minutes after the infusion of OSCS. The third pig showed a transient but pronounced spike in heart rate with a corresponding drop in blood pressure (Fig. 5B). In contrast, none of the three pigs treated with chondroitin sulfate A showed any significant changes in blood pressure or heart rate within the first 30 minutes after drug infusion. Thus, intravenous infusion of OSCS is capable of recapitulating the hallmark cardiovascular features of the reaction in swine. The changes in physiological parameters were mirrored by rapid induction of the amidolytic activity of kallikrein (Fig. 5C). Kallikrein activity remained high throughout the observation period, even after the vital functions returned to normal, suggesting depletion of high-molecular-weight kininogen and inactivation of bradykinin by kininases in vivo, as previously shown with dextran sulfate. ${ }^{20}$ Induction of kallikrein activity was evident in all animals that received OSCS-contaminated heparin, even when no substantive changes in blood pressure were observed. These findings suggest that activation of kallikrein does not always manifest as clinical symptoms, perhaps because of individual variation in control mechanisms that regulate bradykinin activity. Nonetheless, these results also suggest that swine may be an appropriate species in which to assess the potential consequences of OSCS contaminant in cardiovascular and dialysis models as well as in heparin-coated devices.

\section{Discussion}

The recent reports of allergic-type serious adverse events in patients receiving heparin and the subsequent detection of widespread contamination have caused intense international concern about the safety of this essential drug. Urgent problems included an immediate and unknown risk to patients' lives, a threat to the supply of a widely used, essential drug, and the need for international cooperation in managing the integrity of a global supply chain. This crisis necessitated an urgent need to both understand the basis for these clinical events and to prevent future occurrences. The development of an analytic assay for OSCS, coupled with the rapid response of manufacturers and regulatory authorities around the world, has undoubtedly limited the harm. However, in the absence of a biologic link between the OSCS contaminant and the adverse events, the adequacy of screening heparin lots to prevent a recurrence is a concern.

Determining whether a link exists between the presence of OSCS and a biologic response required the convergence of two distinct analyses. First, there was a requirement to develop 
analytic techniques of sufficient sensitivity and specificity to ensure accurate identification and quantification of contaminants or impurities that are present within heparin. Second, there was a requirement to develop a sensitive, clinically appropriate biologic test to determine at what levels, if any, the OSCS would have relevant biologic activity.

With regard to the analytic techniques, a tiered approach was required to ensure effective translation to biologic characteristics. Screening methods were developed to rapidly identify whether heparin lots were contaminated or impure. Then, methods were further developed to enable quantification of the contamination levels. Finally, more sophisticated techniques, such as multidimensional NMR, enabled complete characterization of the contaminant or impurity. This tiered approach was necessitated by the fact that heparin is a polydisperse mixture of glycosaminoglycan chains; orthogonal techniques were therefore required to ensure capture of the other nonheparin components.

Here, we demonstrate that the OSCS present in suspect heparin lots, as well as a synthetic OSCS standard, can directly activate the contact system and induce the generation of C3a and C5a anaphylatoxins in vitro. Moreover, OSCS activates kallikrein in vivo and can induce a profound hypotensive response in pigs, thus providing a potential biologic link between the contaminant and the anaphylactoid reactions seen in affected patients. The finding that hypotension did not develop in all animals treated with OSCS-contaminated heparin, even at a relatively high dose, is consistent with the observation that the majority of patients who received contaminated heparin did not experience an adverse event. However, it is important to note that all animals treated with OSCS-contaminated heparin showed evidence of kallikrein activation in vivo, even in the absence of clinical signs. Patients undergoing dialysis who are also receiving heparin therapy are already at high risk for hypotension because of their exposure to the dialysis membrane, which can also activate the contact system, and their treatment with angiotensin-converting-enzyme inhibitors, which inhibit bradykinin degradation. Exposure to OSCS-contaminated heparin may further increase the risk and could potentially trigger an adverse event. Finally, these findings also suggest that a simple in vitro bioassay could complement the previously described analytic tests $^{4}$ to help protect the global supply chain of heparin, by allowing the screening of heparin lots for the presence not only of OSCS but also of other polysulfated contaminants that may have unintended pharmacologic consequences.

\section{Supplementary Material}

Refer to Web version on PubMed Central for supplementary material.

\section{Acknowledgments}

Supported by grants from the National Institute of General Medical Sciences and the National Heart and Lung Institute (GM57073 and HL080278-01, respectively, to Dr. Sasisekharan).

Drs. Kishimoto, Ganguly, Lansing, Zhao, Galcheva-Gargova, Roy, Shriver, and Venkataraman, Mr. Smith, and Mr. Baily report being employees of Momenta Pharmaceuticals and holding equity in the company, which has technology on the analysis and characterization of complex mixtures, including heparin. Drs. Sasisekharan and Langer report receiving consulting fees from Scientific Protein Labs and Momenta Pharmaceuticals and holding equity in Momenta Pharmaceuticals. Dr. Austen reports receiving consulting fees from Momenta Pharmaceuticals.

We thank Dr. Allen P. Kaplan for helpful discussions; Pete Jobst, Animal Resource Manager, and Andrea Aman, Animal Care Technician, at the Virginia-Maryland Regional College of Veterinary Medicine for their excellent technical help during the in vivo experiments; and Ms. Alison Long, Mr. Chris Honan, Dr. Cedric Hubeau, Mr. Erick Moy, and the staff of the Massachusetts Institute of Technology Division of Comparative Medicine for help with the animal studies. 


\section{References}

1. Acute allergic-type reactions among patients undergoing hemodialysis - multiple states, 20072008. MMWR Morb Mortal Wkly Rep. 2008; 57:124-5. [PubMed: 18256585]

2. Information on adverse event reports and heparin. Rockville, MD: Food and Drug Administration; 2008. at http://www.fda.gov/cder/drug/infopage/heparin/adverse_events.htm [Accessed May 12, 2008]

3. Information on heparin sodium injection. Rockville, MD: Food and Drug Administration; 2008. at http://www.fda.gov/cder/drug/infopage/heparin/default.htm\#screening [Accessed May 12,2008]

4. Guerrini M, Beccati D, Shriver Z, et al. Oversulfated chondroitin sulfate is a major contaminant in heparin associated with adverse clinical events. Nat Biotechnol. in press.

5. Hojima Y, Cochrane CG, Wiggins RC, Austen KF, Stevens RL. In vitro activation of the contact (Hageman factor) system of plasma by heparin and chondroitin sulfate E. Blood. 1984; 63:1453-9. [PubMed: 6202346]

6. Henry SP, Giclas PC, Leeds J, et al. Activation of the alternative pathway of complement by a phosphorothioate oligonucleotide: potential mechanism of action. J Pharmacol Exp Ther. 1997; 281:810-6. [PubMed: 9152389]

7. Silverberg M, Diehl SV. The autoactivation of factor XII (Hageman factor) induced by low-Mr heparin and dextran sulphate: the effect of the Mr of the activating polyanion. Biochem J. 1987; 248:715-20. [PubMed: 2449171]

8. Baxter provides update on the heparin investigation. Deerfield, IL: Baxter; Mar 19. 2008 at http:// www.baxter.com/products/biopharmaceuticals/downloads/heparin_03-19-08.pdf [Accessed May 12, 2008]

9. Silverberg M, Dunn JT, Garen L, Kaplan AP. Autoactivation of human Hageman factor: demonstration utilizing a synthetic substrate. J Biol Chem. 1980; 255:7281-6. [PubMed: 7391081]

10. Verhamme IM, Bock PE, Jackson CM. The preferred pathway of glycosaminoglycan-accelerated inactivation of thrombin by heparin cofactor II. J Biol Chem. 2004; 279:9785-95. [PubMed: 14701814]

11. Stead N, Kaplan AP, Rosenberg RD. Inhibition of activated factor XII by antithrombin-heparin cofactor. J Biol Chem. 1976; 251:6481-8. [PubMed: 61963]

12. Olson ST, Sheffer R, Francis AM. High molecular weight kininogen potentiates the heparinaccelerated inhibition of plasma kallikrein by antithrombin: role for antithrombin in the regulation of kallikrein. Biochemistry. 1993; 32:12136-47. [PubMed: 7692967]

13. Pixley RA, Cassello A, De La Cadena RA, Kaufman N, Colman RW. Effect of heparin on the activation of factor XII and the contact system in plasma. Thromb Haemost. 1991; 66:540-7. [PubMed: 1725067]

14. Kaplan AP, Austen KF. A prealbumin activator of prekallikrein. II. Derivation of activators of prekallikrein from active Hageman factor by digestion with plasmin. J Exp Med. 1971; 133:696712. [PubMed: 4251126]

15. Ghebrehiwet B, Silverberg M, Kaplan AP. Activation of the classical pathway of complement by Hageman factor fragment. J Exp Med. 1981; 153:665-76. [PubMed: 7252410]

16. DiScipio RG. The activation of the alternative pathway $\mathrm{C} 3$ convertase by human plasma kallikrein. Immunology. 1982; 45:587-95. [PubMed: 6916710]

17. Wiggins RC, Giclas PC, Henson PM. Chemotactic activity generated from the fifth component of complement by plasma kallikrein of the rabbit. J Exp Med. 1981; 153:1391-404. [PubMed: 6910482]

18. Schaiff WT, Eisenberg PR. Direct induction of complement activation by pharmacologic activation of plasminogen. Coron Artery Dis. 1997; 8:9-18. [PubMed: 9101117]

19. Wiggins RC. A different cleavage site for high molecular weight kininogen in vivo following intravenous injection of dextran sulfate in the rabbit. Circ Res. 1986; 58:595-604. [PubMed: 2421941]

20. Siebeck M, Cheronis JC, Fink E, et al. Dextran sulfate activates contact system and mediates arterial hypotension via B2 kinin receptors. J Appl Physiol. 1994; 77:2675-80. [PubMed: 7534754] 


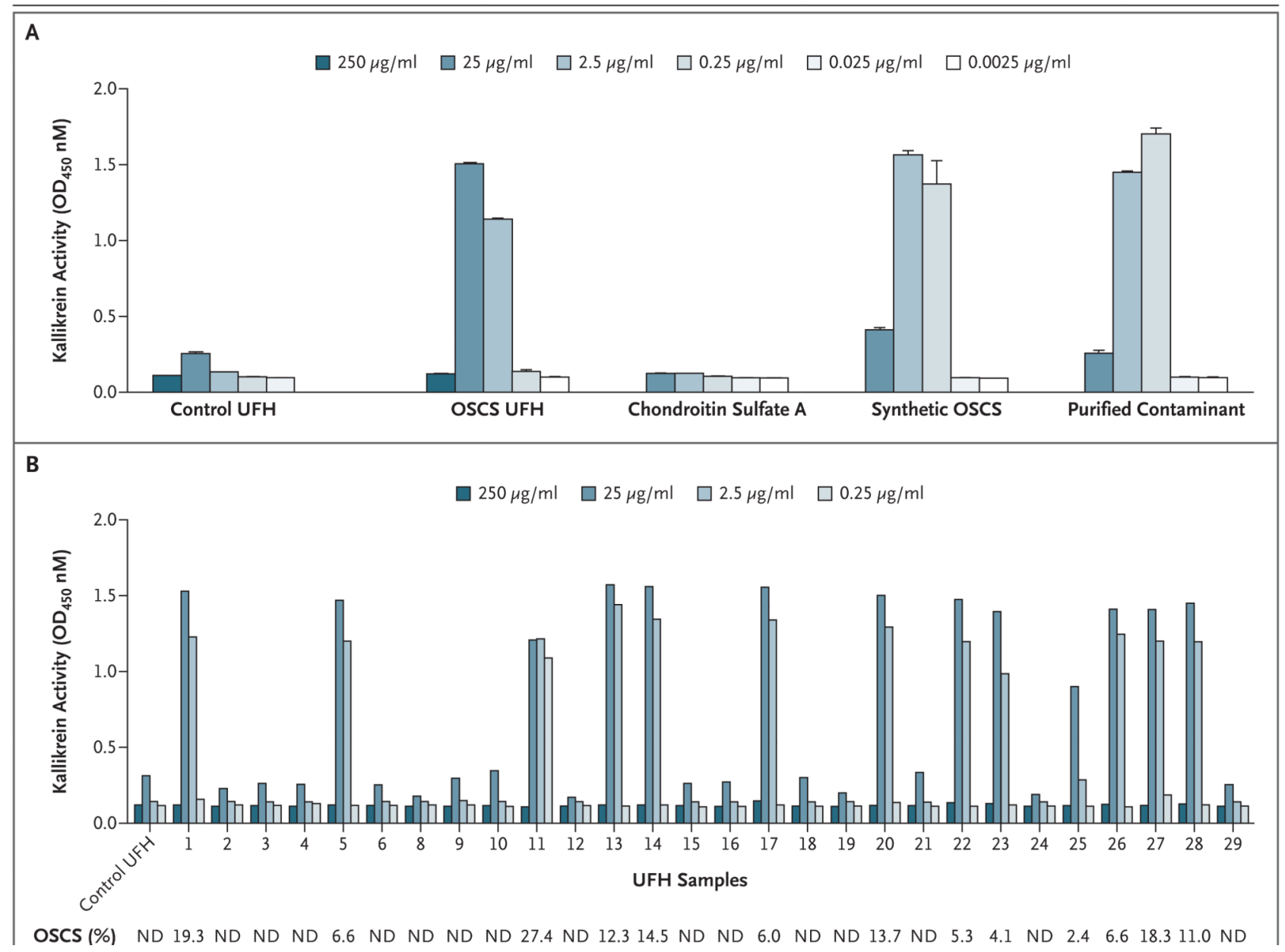

Figure 1. Effect of OSCS on Kallikrein Activity

Pooled human plasma samples were treated with control unfractionated heparin (UFH) or OSCS-contaminated heparin ( 0.025 to $250 \mu \mathrm{g}$ per milliliter) or with chondroitin sulfate A, synthetic OSCS, or purified OSCS contaminant ( 0.0025 to $25 \mu \mathrm{g}$ per milliliter). Amidolytic activity was assessed by the addition of the S-2302 chromagenic substrate (D-Pro-Phe-Arg-pnitroaniline); the effect on kallikrein amidolytic activity is shown (Panel A). The presence of OSCS in heparin was associated with the induction of kallikrein activity. Twenty-nine samples of heparin, representing both suspect heparin lots and control lots, were analyzed in a blinded fashion for both the presence of OSCS and the ability to activate kallikrein (Panel B). The presence of OSCS was detected and quantified by one-dimensional nuclear magnetic resonance spectroscopy (see Figure 2 in the Supplementary Appendix). The percentage of each sample that was OSCS is shown below the plot. Kallikrein amidolytic activity was assessed at various concentrations of heparin; Sample 7 was not analyzed for kallikrein activity owing to the limited quantity available. ND denotes not detectable, and OD optical density. In Panel A, T bars indicate standard deviations of replicate measurements. 


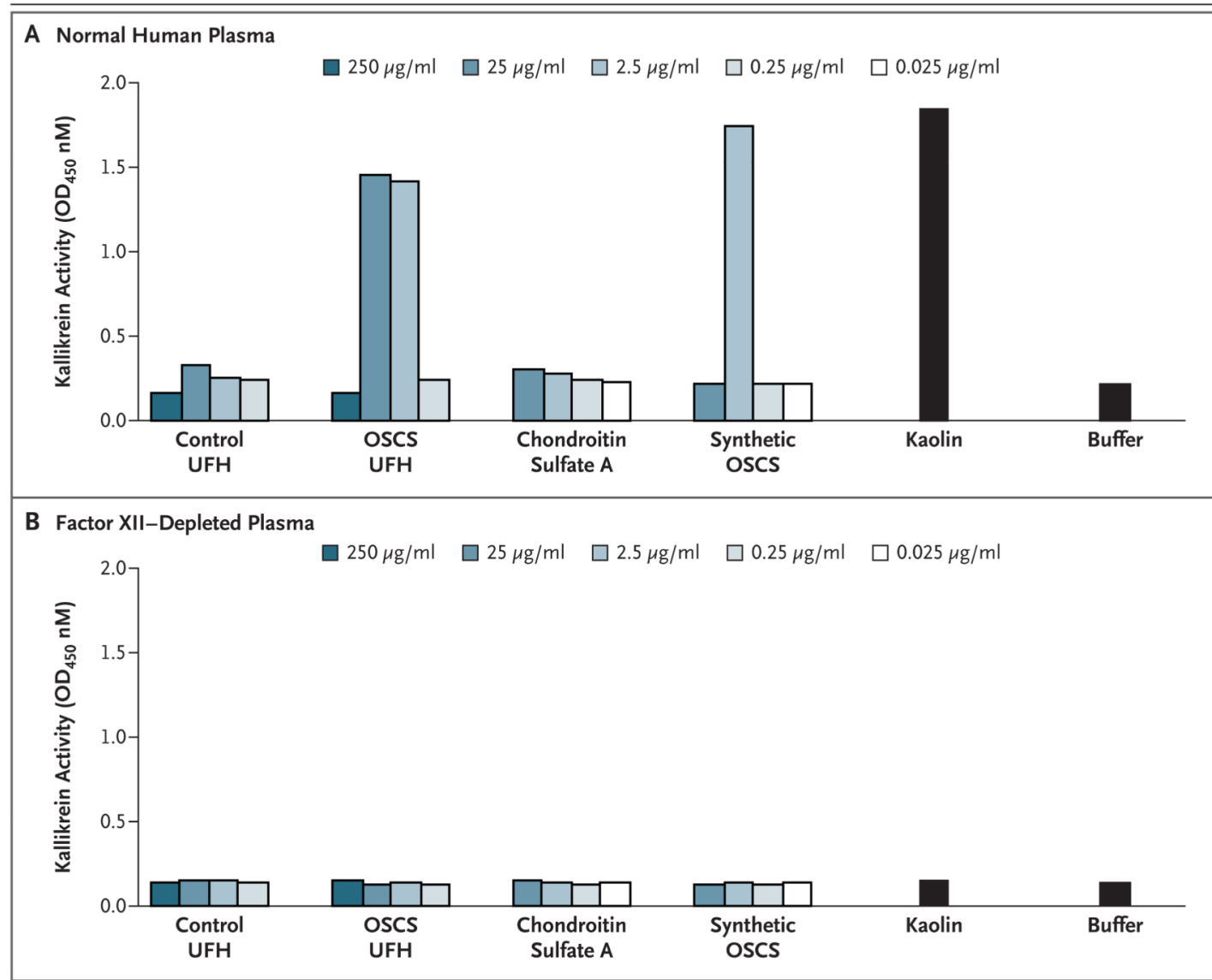

Figure 2. OSCS-Induced Activation of Kallikrein in Normal or Factor XII-Depleted Plasma Control unfractionated heparin (UFH), OSCS-contaminated UFH, chondroitin sulfate A, and synthetic OSCS were incubated with normal plasma (Panel A) or with factor XII-depleted plasma (Panel B); OSCS-induced activation of kallikrein is dependent on factor XII, as indicated. Kaolin-containing buffer was evaluated as a positive control activator of the contact system. Buffer alone was included as a negative control. Amidolytic activity was assessed by the addition of the S-2302 chromagenic substrate (D-Pro-Phe-Arg-pnitroaniline). OD denotes optical density. 

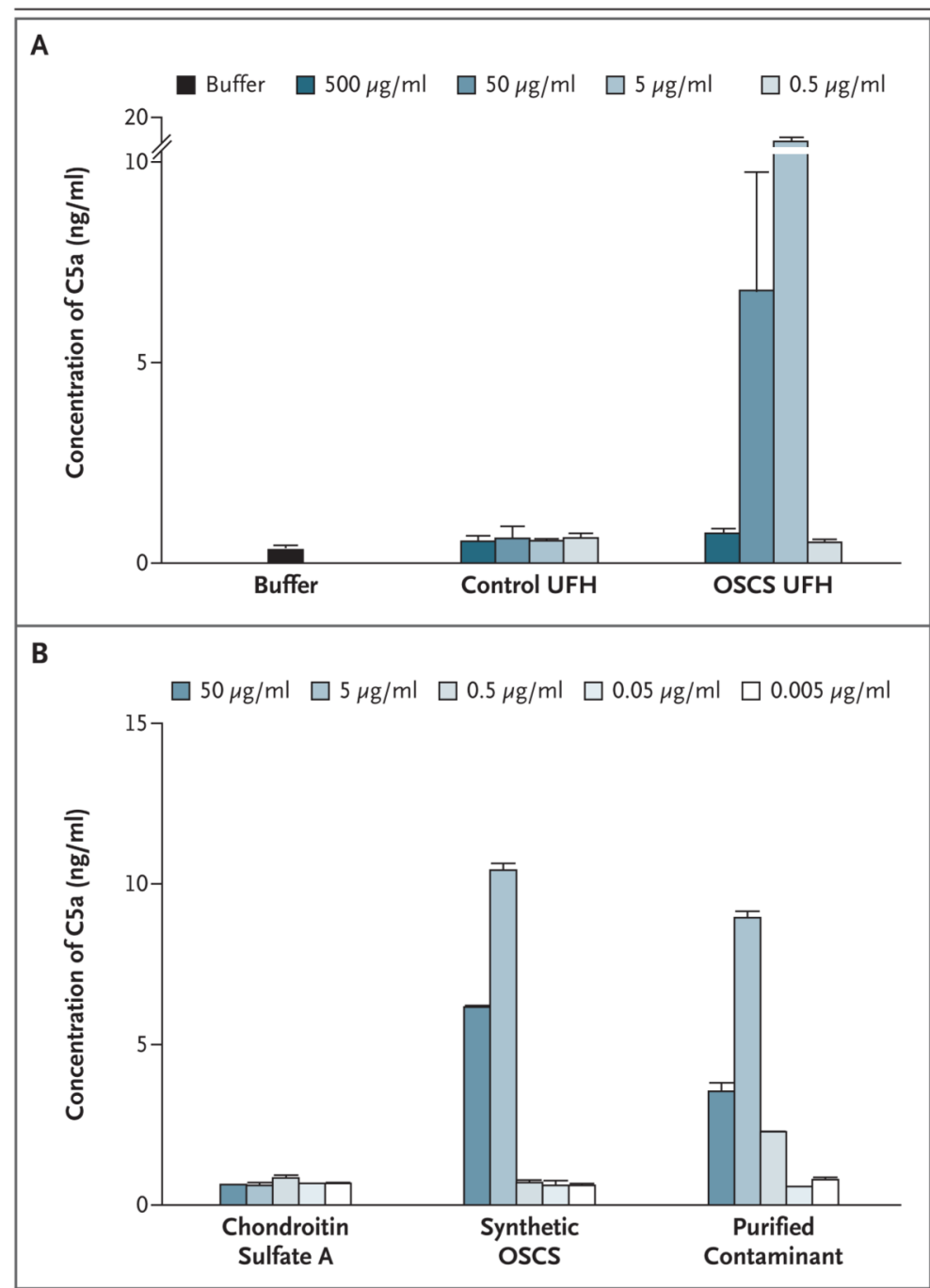

Figure 3. OSCS and the Generation of Complement-Derived C5a Anaphylatoxin in Human Plasma

OSCS-contaminated unfractionated heparin (UFH) and control UFH ( 0.5 to $500 \mu \mathrm{g}$ per milliliter) (Panel A) or chondroitin sulfate A, synthetic OSCS, and purified OSCS contaminant ( 0.005 to $50 \mu \mathrm{g}$ per milliliter) (Panel B) were incubated with normal plasma anticoagulated with EDTA. The generation of C5a was assessed by means of ELISA. T bars indicate standard deviations of replicate measurements. 


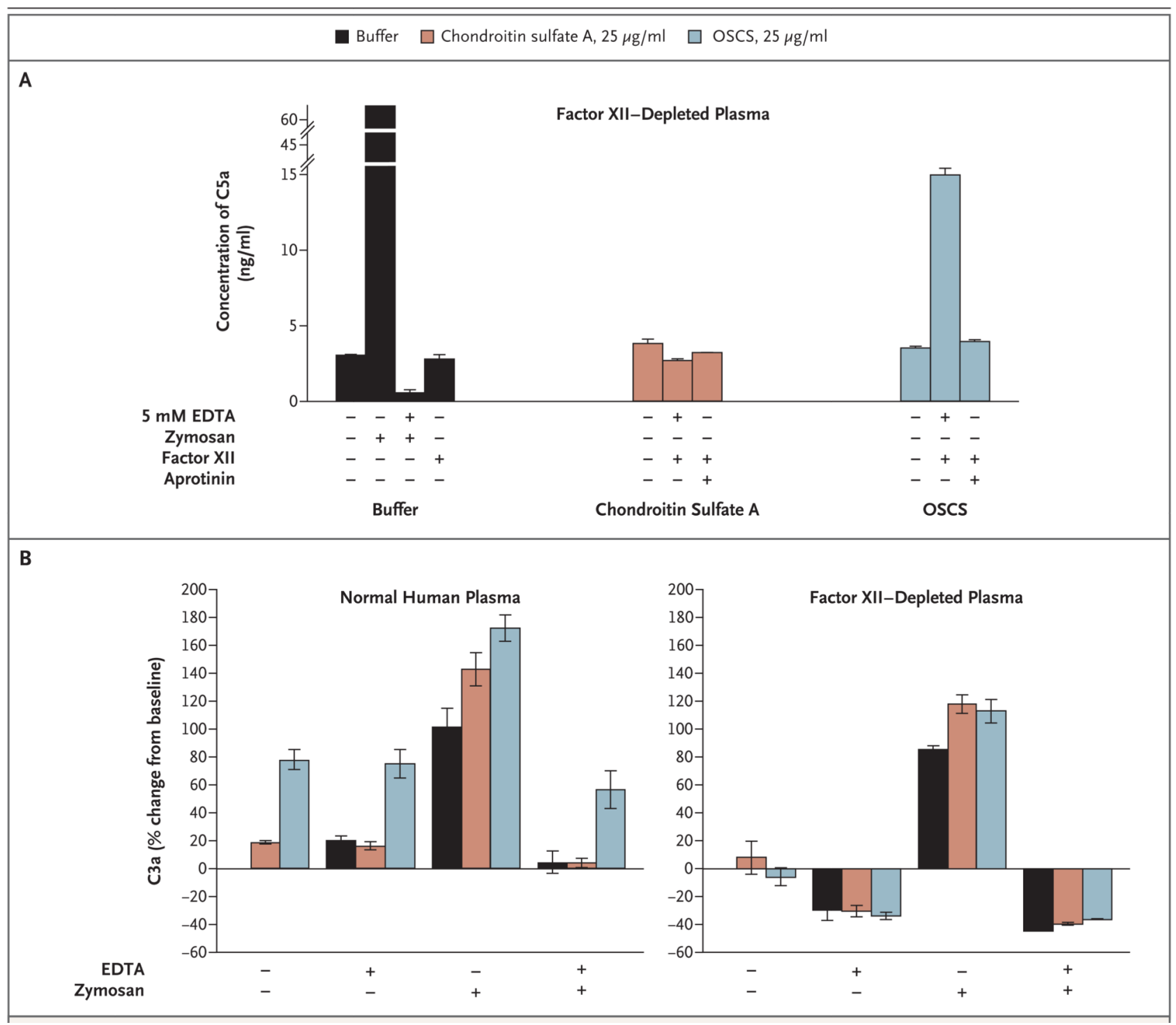

Figure 4. OSCS Induction of Complement-Derived C3a and C5a Anaphylatoxins and Its Relationship to the Contact System

Factor XII-depleted plasma was incubated with chondroitin sulfate A, OSCS, or control buffer in the presence or absence of $5 \mathrm{mM}$ EDTA, zymosan (1 mg per milliliter), or aprotinin (400 IU per milliliter) (Panel A). Specific samples were reconstituted with purified factor XII, as indicated. Normal human plasma or factor XII-depleted plasma was incubated with chondroitin sulfate A, OSCS, or control buffer in the presence or absence of $5 \mathrm{mM}$ EDTA, zymosan (1 mg per milliliter), or both (Panel B). C3a and C5a generation was assessed by means of ELISA. T and I bars indicate standard deviations of replicate measurements. 


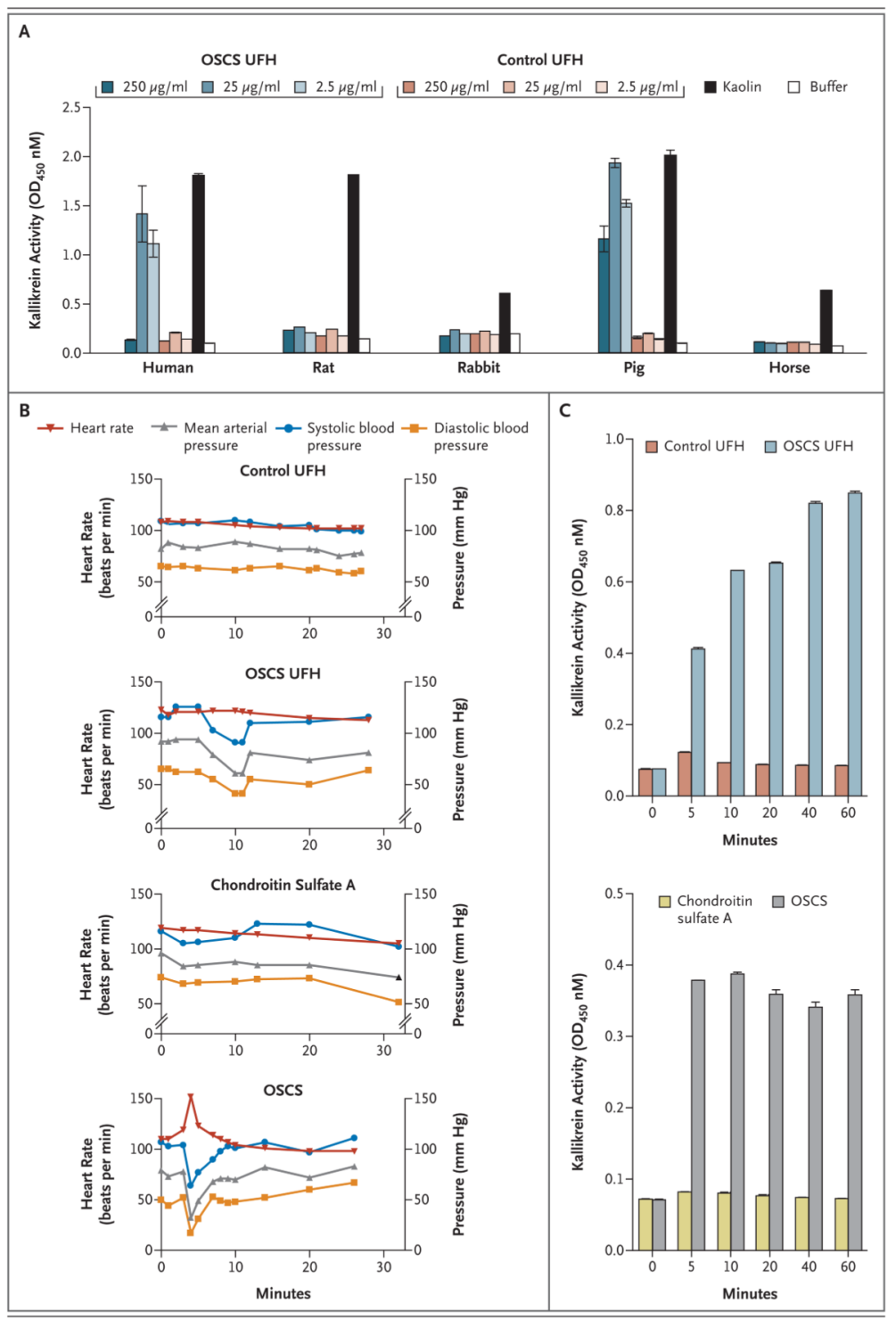

Figure 5. (facing page). In Vitro and In Vivo Activity of OSCS

Human, rat, rabbit, pig, and horse plasma samples were incubated with various concentrations of OSCS-contaminated unfractionated heparin (UFH) or control UFH (Panel A). Kaolin-containing buffer was tested as a positive control. Buffer alone was included as a negative control. Kallikrein amidolytic activity was assessed by the addition of the S-2302 chromogenic substrate; OSCS induces hypotension and kallikrein activity in swine (Panels $\mathrm{B}$ and $\mathrm{C}$ ). Anesthetized Yorkshire crossbred pigs (three to six pigs per group) were treated with a single intravenous bolus (5 $\mathrm{mg}$ per kilogram) of control UFH, OSCS-contaminated UFH, chondroitin sulfate A, or synthetic OSCS. Representative data for the heart rate, the mean arterial pressure, the systolic blood pressure, and the diastolic blood pressure are shown (Panel B). EDTA-anticoagulated plasma was collected at baseline and at 5, 10, 20, 40, and 60 minutes after infusion of test samples (Panel C). OD denotes optical density. In Panels A and C, T and I bars indicate standard deviations of replicate measurements. 